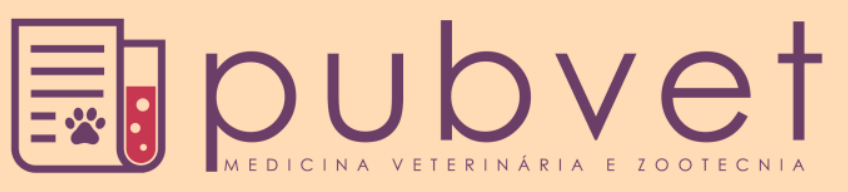

https://doi.org/10.22256/pubvet.v12n2a38.1-4

\title{
Frequência de erros pré-analíticos ocorridos na Medicina Veterinária
}

\author{
Paulo Henrique Braz ${ }^{\emptyset} 1_{*}$, Eduarda Rodrigues Garcia ${ }^{2}$ \\ ${ }^{1}$ Docente do curso de Medicina Veterinária, Centro Universitário da Grande Dourados, Dourados-MS Brasil. E-mail:pauloh.braz@hotmail.com \\ ${ }^{2}$ Acadêmica do curso de Medicina Veterinária, Centro Universitário da Grande Dourados, Dourados-MS Brasil.E-mail: duda_723@hotmail.com \\ *Autor para correspondência.
}

RESUMO. Os exames laboratoriais são de extrema importância para chegar a um diagnóstico definitivo, eles auxiliam em 70\% nas tomadas de decisões médicas. Como em qualquer circunstância, existem probabilidade de erros, que muitas vezes são ocasionadas por negligência ou falta de conhecimento do assunto. O presente trabalho teve como objetivo demonstrar os principais erros pré-analíticos ocorridos na medicina veterinária Os principais erros demonstrados foram relacionados a não utilização de luvas de procedimento, seguido da não realização da tricotomia no local de colheita de sangue. Foi possível averiguar que há conhecimento da parte dos médicos veterinários sobre qual o melhor tubo a ser utilizado na rotina clínica de colheita e envio de sangue ao laboratório, entretanto, ainda há falhas no tempo de garrote. O erros pré-analíticos são procedimentos facilmente reparáveis quando estabelecida uma metodologia para a colheita de material biológico.

Palavras chave: Exames laboratoriais, testes séricos, hemograma, exames bioquímicos.

\section{Frequency of preanalytical errors occurred in Veterinary Medicine}

ABSTRACT. The laboratory tests are extremely important for a definitive diagnosis, they assist in 70\% in medical decision making. As in any circumstance, there are likelihood of errors, which are often occasioned by negligence or lack of knowledge of the subject. The present study aimed to demonstrate the main pre-analytical errors in veterinary medicine. The main errors reported were related to the non-use of procedure gloves, followed by failure to perform the tricotomy at the blood collection site. It was possible to verify that the veterinarians know about the best tube to be used in the clinical routine of harvesting and sending blood to the laboratory, however, there are still gaps in the club time. Preanalytical errors are known as a methodology for a collection of biological material.

Key words: Laboratory tests, serum tests, blood count, biochemical tests.

\section{Frecuencia de errores pre analíticos ocurridos en la Medicina Veterinaria}

RESUMEN. Los exámenes de laboratorio son de extrema importancia para un diagnóstico definitivo, ellos auxilian en un $70 \%$ en la toma de decisiones médicas. Como en cualquier circunstancia, existen probabilidades de errores, que a menudo son ocasionados por negligencia o falta de conocimiento del asunto. El presente trabajo tuvo como objetivo demostrar los principales errores preanalíticos ocurridos en la medicina veterinaria. Los principales errores demostrados se relacionaron con la no utilización de guantes de procedimiento, seguido de no realización de la tricotomía en el lugar de recolección de sangre. Es posible averiguar que hay conocimiento de parte de los médicos veterinarios 
sobre cuál el mejor tubo a ser utilizado en la rutina clínica de recolección y envío de sangre al laboratorio, sin embargo, todavía hay fallas en el tiempo de garrote. Los errores preanalíticos se conocen como una metodología para una cosecha de material biológico.

Palabras clave: Exámenes de laboratorio, pruebas séricas, hemograma, exámenes bioquímicos.

\section{Introdução}

Os exames complementares são de extrema importância para o diagnóstico, utilizados tanto na medicina veterinária quanto na medicina. Contribuem para um diagnóstico específico em $70 \%$ dos casos (Codagnone \& Guedes, 2014). Para a realização dos procedimentos laboratoriais, é necessário que sejam seguidas algumas etapas padronizadas, a fim de garantir maior confiabilidade ao laboratório escolhido e no melhor resultado dos exames solicitados (Soares et al., 2013), levando em conta que um resultado errôneo pode acarretar em falhas terapêuticas (Chaves \& Marin 2010).

Existem três diferentes fases para a realização de um exame, definidas como fase pré-analítica, fase analítica e a fase pós-analítica. Todas estas etapas estão sujeitas a erros, podendo estes acontecer de forma isolada ou simultânea. (BRASIL, 2005).

A fase pré-analítica é a fase que antecede a análise do material biológico e inicia-se na solicitação do exame pelo médico veterinário até a colheita da amostra e transporte. Os erros cometidos nesta primeira etapa varia de 46 a $68,2 \%$ (Oliveira, 2009), já, a fase analítica, corresponde ao processamento do material biológico a ser analisado (Codagnone \& Guedes, 2014), com erros variando entre 7 a 13\% (LimaOliveira, 2009) e por último, os erros da fase pósanalítica, ocasionados por digitação de forma errada ou má interpretação do laudo, ocorre em porcentagem que variam entre 18,5 e $47 \%$, (Andriolo et al., 2014).

Este trabalho tem como objetivo demonstrar os principais erros pré-analíticos ocorridos na medicina veterinária visando auxiliar os profissionais da área quanto à atenção nesta fase, primordial para o sucesso no resultado do exame solicitado.

\section{Material e Métodos}

O trabalho foi aprovado pelo Comitê de Ética na Utilização De Animais - CEUA-UNIGRAN, sob protocolo 028/2016 e parecer consubstanciando. Por um período de um mês, foi acompanhada a rotina de atendimento e internação de animais de uma Clínica Veterinária, localizada no município de Dourados, MS.

Inicialmente foi projetada uma planilha para averiguar os possíveis erros pré-analíticos que poderiam ser cometidos, tais como: Exame solicitado de forma correta, uso de luvas, tricotomia no local de colheita do material, veia escolhida para colheita de sangue, assepsia do local, tempo de garrote, calibre da agulha escolhida, tamanho da seringa, escolha do tubo correto de acordo com o exame a ser solicitado, tempo de refrigeração da amostra, e tempo de realização do exame após a colheita.

Foram acompanhados os procedimentos de 130 atendimentos, com totalidade de 152 exames solicitados de caninos e felinos sem predisposição de idade, sexo, peso ou raça.

\section{Resultados e Discussão}

No total, foram coletados exames de 130 animais diferentes, obtendo-se 152 exames, devido a alguns animais serem submetidos a mais de um tipo de exame. O Gráfico 1 representa em porcentagem a quantidade de exames realizados

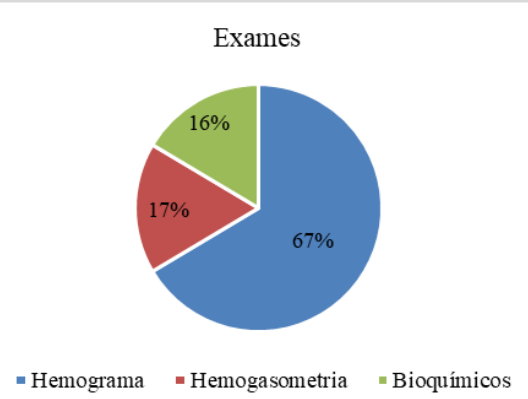

Gráfico 1. Exames coletados nos animais no período de estudo. Foram levados em consideração o hemograma, exames bioquímicos séricos (Alanina Amino transferase, Fosfatase Alcalina, Cratinina, Ureia, Glicose, proteínas totais) e gasometria (arterial e venosa).

A veia jugular foi a via de acesso mais comum para a colheita de sangue para hemograma, bioquímicos séricos e gasometria venosa (Tabela 1), representado por uma frequência de $91,54 \%$. Esta veia para acesso foi escolhida principalmente devido a facilidade de 
colheita em uma veia de maior calibre e com maior fluxo sanguíneo (BRASIL, 2005).

Tabela 1. Principais vias de acesso sanguíneo em cães e gatos para colheita de sangue venoso

\begin{tabular}{lcc}
\hline Local de colheita & Colheitas & $\%$ \\
\hline Veia jugular & 119 & 91,54 \\
Veia femoral & 8 & 6,15 \\
Veia cefálica & 3 & 2,31 \\
\hline
\end{tabular}

Um dos procedimentos importantes, na tentativa de minimizar o risco de causar hemólise no sangue amostrado é a escolha correta do calibre da agulha, o volume da seringa e o tubo correto de acordo com o exame a ser realizado. A Tabela 2 demonstra quais foram os principais materiais utilizados para esta finalidade. Quando utilizado o termo "sem necessidade" no tópico referido ao tipo de tubo, se deve aos exames de hemogasometrias. Estes exames foram realizados no próprio laboratório da clínica veterinária, não necessitando da utilização de tubos, apenas uma seringa de $1 \mathrm{~mL}$ com uma quantidade de $1 \mathrm{cc}$ de heparina.

Tabela 2. Frequência na escolha dos materiais para colheita de sangue arterial e venoso

\begin{tabular}{lr}
\hline Modelo da Agulha & Quantidade \\
\hline $25 \times 0,70 \mathrm{~mm}$ & 26 \\
$25 \times 0,80 \mathrm{~mm}$ & 104 \\
$12 \times 0,38 \mathrm{~mm}$ & 1 \\
\hline Seringa & 18 \\
\hline $1 \mathrm{ml}$ & 92 \\
$3 \mathrm{ml}$ & 20 \\
$5 \mathrm{ml}$ & \\
\hline Tubo & 101 \\
\hline Roxo (EDTA) & 22 \\
Vermelho (Tubo seco) & 3 \\
Cinza (Na2EDTA) & 26 \\
Não usou &
\end{tabular}

Em nenhum caso houve confusão ou desconhecimento do clínico veterinário quanto ao tubo a ser utilizado e qual o anticoagulante escolhido nesta circunstância desejada.

Segundo Andriolo et al. (2010a) para realização das hemogasometrias, deve ser usado uma seringa preparada com heparina de lítio com balanceamento de cálcio, pode ser usado a seringa com preparação caseira, usando heparina líquida, porém nesse caso há baixa concentração de sódio, na qual aumenta a possibilidade de aumento do cálcio iônico, resultando em valores falsamente baixos comparados com os valores reais. $\mathrm{Na}$ clínica veterinária foram utilizadas apenas as preparações caseiras, e com isso os exames podem ter tido resultados alterados.

Para fazer o garrote/torniquete, usa-se uma das mãos ou um manguito de borracha para aumentar o fluxo intravascular, porém uma inadequada execução faz com que ocorra alterações no exame e também faz com que haja hematomas no paciente. $\mathrm{O}$ tempo de torniquete também influencia no resultado dos exames, quando se passa mais de 60 segundos, ocorre hemólise da amostra, podendo elevar o nível de potássio e cálcio (Andriolo et al., 2010b, Costa \& Moreli 2012, Lima-Oliveira et al., 2011). Foi possível verificar que em 122 colheitas de sangue foi conseguido realizar na primeira tentativa, 2 casos na segunda tentativa e 6 casos na terceira tentativa. $\mathrm{O}$ tempo de garrote foi representado no gráfico 2.

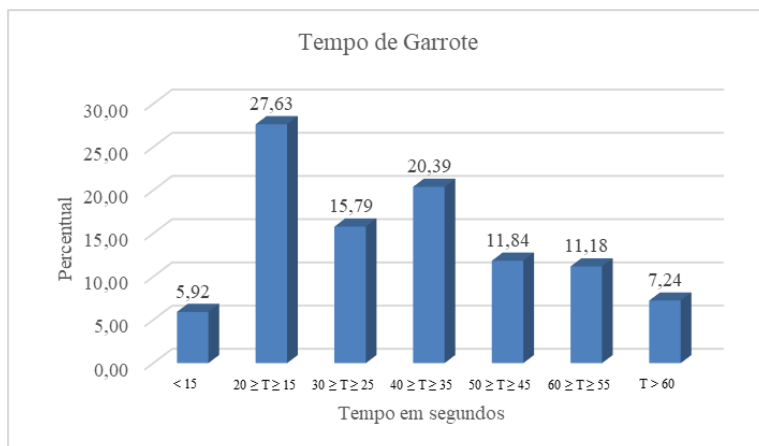

Gráfico 2. Porcentagem referente ao tempo de garrote realizado nos animais para colheita de sangue venoso.

Por último, o flebotomista deve estar paramentado com luvas para diminuir a contaminação da amostra na hora da coleta, o local escolhido para a coleta deve estar limpo, no caso de animais, deve ser realizada a tricotomia do local, que é a retirada do pelo, deve ser feito a assepsia com álcool $70 \%$ e esperar o mesmo secar sozinho, devido que se o lugar estiver úmido, ocorre hemólise do sangue (Andriolo et al., 2010b).

O Gráfico 3 demonstra que o maior erro cometido foi a não utilização das luvas, com totalidade de $85,38 \%$, seguido da não realização da tricotomia $(27,7 \%)$, permanência de umidade no local a ser colhido sangue $(26,9 \%)$ e por fim, não realizar assepsia no local de colheita $(8,5 \%)$.

Após coletado o exame, o mesmo deve ser corretamente identificado para que não ocorram trocas de amostras entre diferentes pacientes (Cenci et al., 2011, Costa \& Moreli, 2012, Lopes 
2003). Houve uma frequência de $9 \%$ de tubos não identificados ao ser encaminhado ao laboratório de análises clínicas.

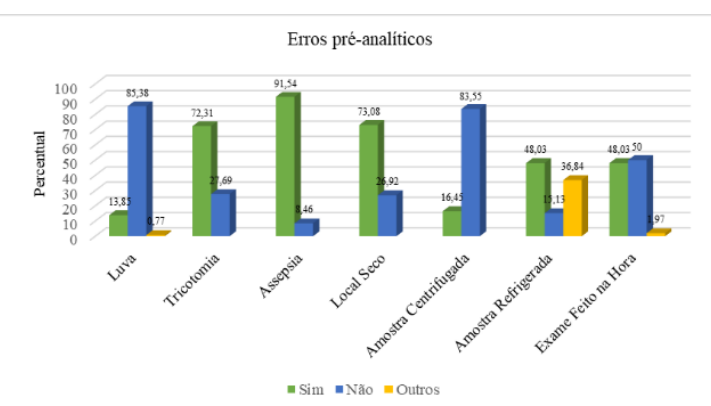

Gráfico 3. Principais erros pré-analíticos ocorridos no local de estudo.

\section{Conclusão}

Diante dos resultados obtidos, o maior erro na fase pré-analítica consiste em não serem utilizadas luvas de procedimento, seguido da insuficiência na realização de tricotomia no local de colheita. $\mathrm{O}$ erros pré-analíticos são procedimentos facilmente reparáveis quando estabelecida uma metodologia para a colheita de material biológico.

\section{Referências Bibliográficas}

Andriolo, A., Faulhaber, A. C. L., Junior, A. P., Martins, A. R., Machado, A. M. O., Ballarati, C. A. F. \& Soares, C. A. S. 2014. Recomendação da sociedade brasileira de patologia clínical medicina laboratorial: coleta e preparo da amostra biológica. 1. ed. Editora Manole, Barueri, SP, Brasil.

Andriolo, A., Martins, A. R., Ballarati, C. A. F., Barbosa, I. V., Mendes, M. E., Melo, M. R., Sumita, N. M., Romano, P. \& Trindade, P. A. 2010a. Recomendação da sociedade brasileira de patologia clínica medicina laboratorial para coleta de sangue venoso. 2. ed., Editora Manole, Barueri, SP, Brasil.

Andriolo, A., Martins, A. R., Machado, a. M. O., Ballarati, C. A. F., Galoro, C. A. O., Barbosa, I. V., Martins, L. E. R., Mendes, M. E., Bazanelli, M. G., Melo, M. R., Sumita, N. M., Slhessarenko, N., Romano, P., Aoki, R. N. \& Shcolnik, W. 2010b. Gestão na fase préanalítica, recomendação da sociedade brasileira de patologia clínica/medicina laboratorial. 1. ed., Grafitto Gráfica e Editora, Brasil.

BRASIL. Resolução RDC no. 302, de 13 de Outubro de 2005. Aprova o "Regulamento Técnico Para Laboratórios Clínicos" constante do anexo desta Resolução. Órgão emissor: ANVISA - Agência Nacional de Vigilância Sanitária. Disponível em: http://portal.anvisa.gov.br/documents/10181/2 718376/RDC_302_2005_COMP.pdf/7038e85 3-afae-4729-948b-ef6eb3931b19. [Acesso: 16 out 2017]

Cenci, A., Braga, A. C., Brito, B. G., Cerva, C., Mayer, F. Q., Karam, F. S. C., Martins, J. R. S., Neto, J. A. S. M. 2011. Manual de coleta e remessa de amostras para diagnóstico laboratorial veterinário. n.20. FEPAGRO, Porto Alegre, RS, Brasil.

Chaves, J. S. C. \& Marina, V. A. 2010. Avaliação do controle externo da qualidade nos laboratórios clínicos do Rio de Janeiro de 2006 a 2008. Rio de Janeiro. Jornal Brasileiro de Patologia $e$ Medicina Laboratorial, 46, 391-394.

Codagnone, F. T. \& Guedes, S. S. 2014. Buscando a eficiência laboratorial por meio de indicadores de qualidade: ênfase na fase préanalítica. Rio de Janeiro. Revista ACRED, 4, 27-41.

Costa, V. G. \& Moreli, M. L. 2012. Principais parâmetros biológicos avaliados em erros na fase pré-analítica de laboratórios clínicos: revisão sistemática. Rio de Janeiro. Jornal Brasileiro de Patologia e Medicina Laboratorial, 48, 163-168.

Lima-oliveira, G. S., Picheth, G., Sumita, N. M. \& Scartezini, M. 2009. Controle da qualidade na coleta do espécime diagnóstico sanguíneo: iluminando uma fase escura de erros préanalíticos. Rio de Janeiro. Jornal Brasileiro de Patologia e Medicina Laboratorial, 45, 441-447.

Lima-oliveira, G., Barcelos, L. F., Corrêa, J. A., Guimarães, J. C., Neufeld, P. M. \& Grinberg, I. 2011. Gestão de qualidade na fase préanalítica para 1: análise crítica do CLSI H3-A6. Rio de Janeiro. Revista Brasileira de Análises Clínicas, 46, 85-88.

Lopes, H. J. J. 2003. Garantia e controle da qualidade no laboratório clínico. Gold Analisa Diagnótica, Belo Horizonte, MG, Brasil.

Soares, B. F., Cordeiro, P. P., Sales, B. B. \& Santos, C. F. 2013. Estudo comparativo entre o hemograma humano e veterinário. São Paulo. Ensaios e Ciência: Ciência Biológicas, Agrárias e da Saúde, 16, 87-100.

Article History:

Received 16 October 2017

Accepted 11 November 2017

Available online 27 January 2018

License information: This is an open-access article distributed under the terms of the Creative Commons Attribution License 4.0, which permits unrestricted use, distribution, and reproduction in any medium, provided the original work is properly cited. 